\title{
A geometrical note on the Binary Cubic Form
}

\author{
By T. ScotT
}

(Received 8th January, 1947. Read 17th January, 1947.)

\section{Introduclion.}

1. An extensive investigation into the geometry associated with the binary cubic form as representing a triad of points on a twisted cubic curve has been carried out by Sturm. ${ }^{1}$ The object of the present note is to derive two further geometrical properties, one associated with the twisted cubic, and the other with the rational plane cubic curve.

Following Sturm's treatment I shall take $U$ to be a binary cubic representing three points $A, B$ and $C$ on a twisted cubic, the plane $p$ joining them being the null plane of $P$, the point of intersection of the osculating planes at the three points, with respect to, the fundamental linear complex $N$, viz.,

$$
p_{14}=3 p_{23}
$$

A brief summary of the results required in the present treatment is as follows:-

I. The Hessian $H$ comprises the points $H_{1}, H_{2}$ which are the extremities of the unique chord through $P$.

II. The Cubicovariant $J$ denotes the triad of points $A^{\prime}, B^{\prime}, C^{\prime}$ lying in the plane $q$ which is the harmonic conjugate of $p$ with respect to the osculating planes at $H_{1}, H_{2}$. (Dually, if $Q$ is the null point of $q$ with respect to $N$, then $P Q, H_{1} H_{2}$ are harmonic pairs).

III. The first polar of any point $O$ on the curve with respect to. $U$. represents two points the tangents at which meet the line $O P$, and as $O$ varies these points determine an involution, the chords joining corresponding pairs forming one system of generators of a quadric, which, for convenience, I shall label $S$. Furthermore, since the first polar of $A$, for example, consists of the points $A, A^{\prime}$, the lines $A A^{\prime}$, $B B^{\prime}$ and $C C^{\prime}$ are generators of $S$, as are the tangents at $H_{1}$ and $H_{2}$.

\footnotetext{
- Journal fïr Mathematik, 86 (1879), 121-133.
} 
A Polarity associated with the quadric $S$.

2. It will now be proved that $p$ and $q$ are the polar planes of $Q$ and $P$ respectively with respect to the quadric $S$.

From III of $\S 1$ the three planes $P A A^{\prime}, P B B^{\prime}$ and $P C C^{\prime}$ contain the tangents to the twisted cubic at $A^{\prime}, B^{\prime}$ and $C^{\prime}$ respectively, and since $A A^{\prime}, B B^{\prime}$ and $C C^{\prime \prime}$ are generators of $S$ these three planes are therefore tangential to $S$ at $A^{\prime}, B^{\prime}$ and $C^{\prime}$. That is, the enveloping cone from $P$ must have contact with $S$ in a conic which passes through $A^{\prime}, B^{\prime}$ and $C^{\prime}$. The plane $q$ is therefore the polar plane of $P$ with respect to $S$. Similarly, $p$ is the polar plane of $Q$. This polarity will obviously hold for all involutory pairs of planes given by the pencil of triads $U+\lambda J$, the lines $P Q$ and $p q$ (which are conjugate with respect to the null system $N$ ) also being conjugate with respect to the quadric $S$, while the linear complex $N$ will be self-conjugate with respect to $S$.

An alternative proof can be developed with equal simplicity from a consideration of II of $\S 1$, but it is considered that the above method is more in keeping with the following extension of the theory.

\section{Application to the Rational Plane Cubic Curve.}

3. By projecting the three-dimensional configuration from the point $P$ on to a plane the twisted cubic becomes a rational plane cubic curve, and we obtain the known results ${ }^{1}$ that the double point $H$ is the Hessian of the fundamental triad $U$ which now consists of the three collinear points of inflexion, the cubicovariant $J$ giving the three points of contact of the remaining tangents from the points of inflexion.

The following additional property, however, arises:-

Since the enveloping cone from $P$ meets $S$ in a conic such that the tangent planes through $P A^{\prime}, P B^{\prime}$ and $P C^{\prime}$ touch both the conic and twisted cubic at $A^{\prime}, B^{\prime}$ and $C^{\prime}$ respectively, by projection there will be a tri-tangent conic to the rational plane cubic, the points determined by $J$ being the three points of contact. Moreover, since $p$ is the polar plane of $Q$ with respect to $S$, and $Q$ is projected into the double point of the plane cubic curve, while $p$ becomes the line of inflexions, therefore the line of inflexions is the polar line of the double point with respect to the tri-tangent conic.

1 Grace and Young, Algebra of Invariants, Ch. XI (where the results are derived by means of the Theory of Apolarity).

DUmfries Academy. 